\title{
Amor medicabilis herbis
}

Un essai au XVIII ${ }^{\mathrm{e}}$ siècle de se guérir de l'érotomanie par la Ciguë

Par Gerhard Rudolph

Le Ciel aurait-il fait cet amas de merveilles

Pour la demeure d'un serpent?

Corneille, Psyché, 1671

\section{Le récit}

Lorsqu'en 1784 parurent les Euvres de Valentin Jamerey-Duval (1695-1775) ${ }^{18}$, imprimées par J. J. Thourneysen fils à Bâle, surtout une des illustrations était faite pour éveiller la curiosité du lecteur. Cette vignette (Fig.) se trouvant à la tête des «Lettres à Anastasie Socoloff» représente «Duval tourmenté de la Cigue dont il prit une dose trop forte pour s'empêcher d'être subjugué par l'amour. Un papier étalé sur la table offre le passage de St.Jérôme qui l'engagea à se servir de l'antidote.» Ainsi l'Explication des figures.

La plupart des illustrations de l'ouvrage dédié à l'impératrice Cathérine II de Russie (1729-1796), sont signées Guerin. Sans doute s'agit-il de Christophe Guérin (1758-1831), peintre et graveur (aussi graveur de médailles) à Strasbourg, formé à Paris, et lauréat (1778) de l'Ecole des Beaux-Arts. Il a acquis du renom par la qualité de ses gravures reproduisant des peintures de maîtres anciens, ainsi que par ses illustrations, p. ex. pour «L'homme des champs» de Jacques Delille (Bâle et Strasbourg 1800).

Les vignettes illustrant les écrits posthumes de Jamerey-Duval, ancien directeur de la Bibliothèque et du Cabinet impérial des Médailles à Vienne, publiés par les soins de son ami Von Koch (n1), ont été exécutées par Guérin d'après une suite de neuf estampes que Duval avait fait graver à Vienne par Salomon Kleiner (v.1700-1761) (n2) «pour rappeler les événements principaux de sa vie». Produites sous l'ancien régime, ces vignettes sont des exemples de «gravures d'interprétation», dont Hippolyte Taine disait «rien n'enseigne mieux l'histoire». Maniée avec maitrise par les burinistes de l'Ecole de Paris, l'image était devenue «indispensable pour faire comprendre une pensée, pour créer un mouvement d'opinion en matière politique, religieuse ou sociale». ${ }^{2}$

Ces témoignages se situent loin des fêtes de l'insouciance d'un siècle élégant et frivole qu'on ne s'est pas lassé d'évoquer en parlant de son style. Même ces illustrations modestes de KleinerGuérin pourraient, à mon avis, servir comme preuve pour le postulat de Jean Starobinski ${ }^{32}$ qu'il faut libérer ce siècle de sa légende, et contribuer à regarder son art d'un œil plus attentif suivant le passionnant enseignement de «L'invention de la liberté».

Un passage dans la lettre du 15 juillet 1763 à Anastasie Socoloff se rapporte à la scène présentée par le buriniste: «Lisez, je vous prie la petite 


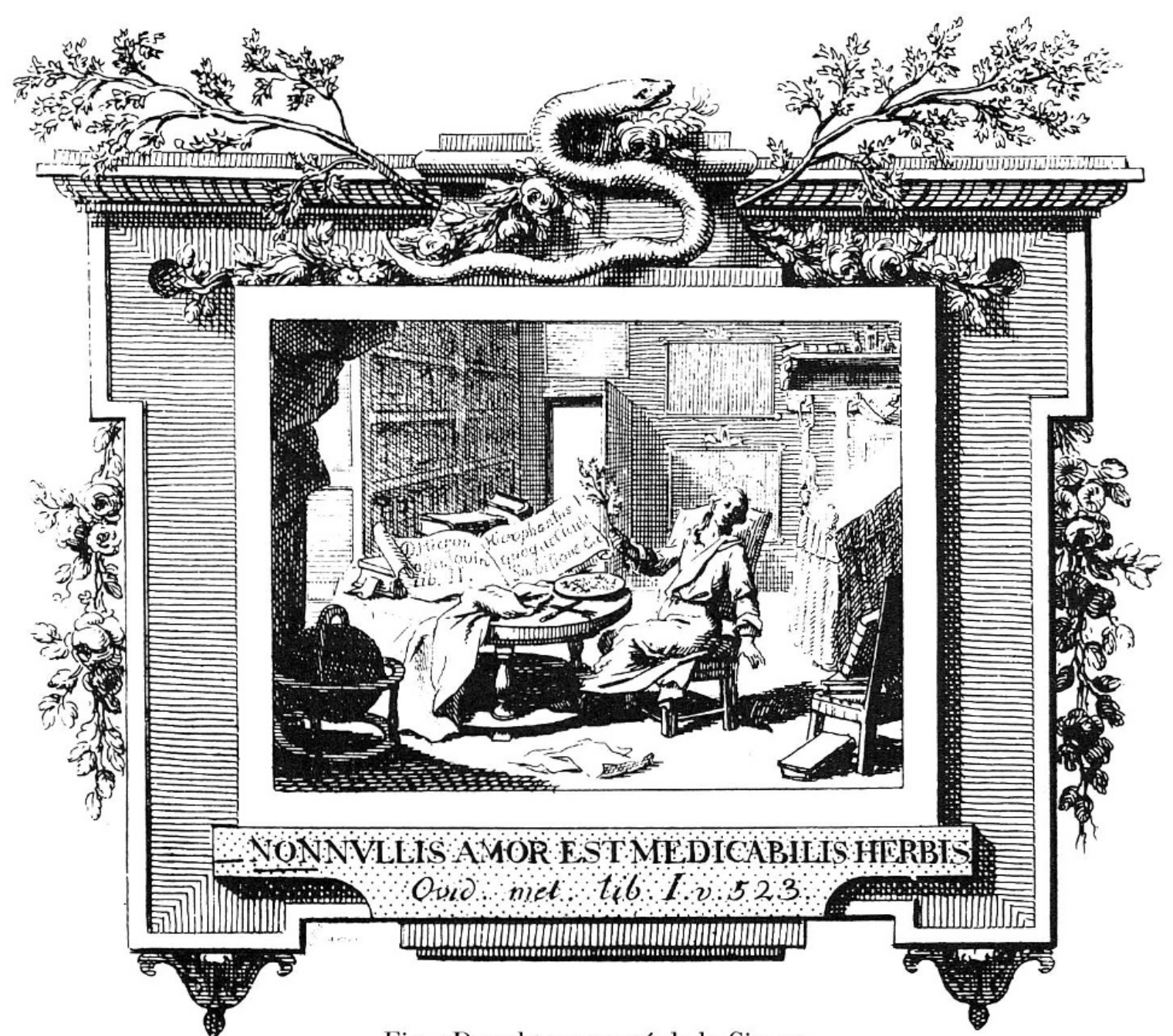

Fig. «Duval tourmenté de la Cigue»

Vignette par C. Guérin (int. $38 \times 53 \mathrm{~mm}$ ), in Duval «EEuvres» 1784

feuille ci-jointe et vous serez au fait de cette aventure, et de ce qui est représenté dans une des petites estampes que vous avez agréées à votre départ de Vienne. S'il vous arrive jamais de vous trouver dans les mêmes angoisses où j'ai été, je vous conjure, aimable Bibi, de ne pas recourir au même remède dont je me suis servi sur la foi de Saint Jérôme. En vérité cette sorte d'antidote est trop violente. Il est glorieux de réfréner les passions quand elles sont trop impérieuses, mais on risque infiniment de vouloir les détruire, je le sais par expérience.» Le soupir d'Apollon, tombé amoureux de Daphné ${ }^{23}$,

«Hei mihi! quod nullis amor est medicabilis herbis:

Nec prosunt domino, quae prosunt omnibus, artes»

est, en partie, entré dans le cartouche. 
Dans les «Mémoires sur la vie de M. Duval» précédant l'édition des CEuvres (1784), Von Koch explique l'événement qui, probablement, eut lieu en 1718, quand Duval avait juste vingt-deux ans: «La vie sédentaire, à laquelle il se livra sans réserve, n'étant plus balancée comme ci-devant par des fréquents exercises du corps, ne tarda pas d'altérer sa constitution. Ses esprits animaux, échauffés par les veilles aussi bien que par une application continuelle, jettèrent du trouble dans son imagination, et ce fut dans cet état que l'aspect fortuit d'une jeune beauté embrasa dans son cœur un amour violent et subit. Fatigué par les combats réitérés que lui livrait la plus fougueuse des passions, il lut un jour dans St. Jérôme, que la cigue était un antidote assuré contre elle. Charmé de cette importante découverte, il se fait apporter sur le champ une bonne quantité de cette herbe et la mange en salade. Cette imprudence pensa lui coûter la vie; le venin ayant glacé son sang et desséché sa poitrine, lui occasionna une dangereuse maladie, dont il ressentit longtemps après les funestes effets. Quelque affaiblie que fut sa santé, ses études n'en souffrirent point...»

Cette expérience avait si profondément marqué Duval qu'il l'a rappelée à plusieurs reprises ${ }^{18,19}$ (n 3$)$. Il a même fait connaître le récit de sa mésaventure à Jean-Jacques Rousseau par l'intermédiaire d'Alexandre Deleyre (1726-1797) qui, en 1759, donne au Citoyen de Genève cette caractéristique: «... un homme non moins rare en son espèce... qui de la garde des brebis qu'il avait dans son enfance est parvenu à celle des livres et médailles de l'Empereur. Il a lui-même écrit son histoire pour son amusement et son instruction» ${ }^{19}$.

Le texte adressé à Rousseau correspond au manuscrit de la Bibliothèque de l'Arsénal à Paris, récemment publié par Jean Marie Goulemot ${ }^{19}$. Ce document autobiographique livre les détails de son étonnante histoire(n 4). Après des réflexions sur la «beauté momentanée, artificielle et plâtrée» des femmes, l'auteur avoue: «Comme je ne les connaissais que très superficiellement, il n'est pas extraordinaire que je me trompasse dans le jugement que je portais d'elles et que je n'eusse encore éprouvé le pouvoir et l'ascendant que la nature ou la coutume leur a donné sur notre cœur.» Peu après, en présence d'une véritable beauté d'à peu près dix-huit ans, fille d'un riche bourgeois de Pont-à-Mousson: «je crus voir Hébé ou Psiché en personne».

D'un moment à l'autre il ne se reconnaissait plus: «J'étais inquiet, sombre, mélancolique, distrait. La retraite, dont auparavant je faisais mes délices, m'ennuyait. La lecture, qui était ma passion dominante, me devenait insipide... Toutes les facultés de mon âme n'étaient remplies que de 
l'image de ma belle que mon imagination avait soin d'orner de toutes les perfections possibles...» A la quatrième et dernière rencontre, invité de s'asseoir auprès d'elle, son émotion fut telle que, pris par un violent tremblement, le malheureux se vit obligé de quitter la compagnie.

A la retraite, il se mit à analyser son propre cas à l'aide du traité sur «les Passions de l'âme» (1649) de Descartes, tout en regrettant de ne pas posséder les connaissances anatomiques de ce savant. A l'article de l'amour sa passion «cessa ses déguisements». La première chose qu'il fit, fut d'abjurer une passion «inutile», même «nuisible et préjudiciable». «Cette résolution ne fut pas l'effet de ma haine pour une passion également séduisante et naturelle. J'étais en âge de la ressentir mais nullement en situation de m'y livrer, et de la satisfaire. Je me représentais que l'on m'avait tiré de l'obscurité des forêts et envoyé au Pont à Mousson pour y étudier, y acquérir de la science et des lumières et point du tout pour y être amoureux et $\mathrm{y}$ faire le joli cœur...»

«Cette guerre intestine (entre esprit et cœur) dura des semaines entières... Le feu qui me consumait et l'ardeur qui circulait dans mes veines me rendirent à la fin tellement insupportable à moi même que j'en perdis l'appétit et le sommeil. Je n'oubliais rien pour me distraire et ralentir le feu qui me dévorait... Mais j'avais beau faire, l'ennemi que je fuyais était en moimême et il n'était pas possible de m'éviter.»

Le recours à des procédures de mortification et de flagellation («cette pieuse et sanglante exécution») augmentait l'agitation de telle sorte «que je crus que tous les feux de l'enfer étaient dans mon cœur... Ma situation me parut si cruelle que je fus tenté de croire qu'elle n'était pas... naturelle et que le diable en était l'instigateur». Le Commentaire de Saint Bruno d'Ast (XII siècle) glosant sur un passage de Job («sub umbra dormit Behemot in secreto calami et in locis humentibus») lui donne la conviction que se sont les parties génitales où s'installe ce que Bruno appelait le Diable: «in his... diabolus dormit, in his delectatur, et quiescit; hoc semper sollicitat et movet.»

«...il me semblait véritablement que l'esprit malin avait minuté ma perte par la résolution qu'il me suggéra. Car voyant que mes efforts et tout ce que j'avais pu faire n'avait rien produit, je me souvins que Descartes avait expliqué d'une manière fort mécanique la passion dont j'étais agité, de façon qu'il la faisait consister plutôt dans la disposition du sang que dans celle de l'esprit. Ses idées à cet égard me firent penser que si je trouvais le moyen de changer la disposition actuelle de mon sang, que peut-être la passion qui me dominait changerait aussi.»

«... en attendant que je trouvasse ce moyen, j’allai dans la Bibliothèque... 
lorsque un malheureux hazard me fit tomber sur ce que dit saint Jérôme à la fin de son Premier livre contre Jovinien (393) (n5) des hiérophantes ou prêtres de Cérès d'Athènes...: jusqu'à son temps ces prêtres, obligés par leur fonction de garder la continence, se servaient pour cet effet du jus de ciguë («Hierophantes quoque Athenensium usque hodie cicutae sorbitione castrari; et postquam in pontificatum fuerint allecti, viros esse desinere»). Cependant, «saint Jérôme n'avait pas jugé à propos de détailler la dose ou quantité de cigue que ces prêtres prenaient pour produire l'effet qu'ils espéraient. Or mon intention était bien d'éteindre en moi les ardeurs de la concupiscence en domptant et en affaiblissant les vivacités de mon tempérament, mais je ne prétendais nullement subir le sort de Socrate...»

Dans un jardin en friche «entre la Moselle et le mur extérieur du collège», où poussait la cigue, «j’en cueillis une petite poignée de la plus verte et de la plus succulente et lorsque l'heure du souper fut arrivée, j'en mêlai une quantité de feuilles, que je crus proportionnée au besoin que j'en avais, avec la portion de salade que l'on avait coutume de me donner»... Ressentissant «toute l'horreur de mon action... je me livrai à un assoupissement auquel l'accablement avait beaucoup plus de part que le sommeil. M'étant réveillé environ à une heure après minuit, je fus étrangement surpris de me trouver un tout autre homme que je n'avais été quatre ou cinq heures auparavant. Le charme des passions était évanoui, le masque et le bandeau de l'amour tombé, et l'idée de ma belle tellement affaiblie dans mon imagination que je me souvins d'elle comme j'aurais fait de toute autre personne de ma connaissance. Ainsi mon cœur se trouva tout à coup dégagé, guéri de ses blessures, mais je ne tardai pas à m'apercevoir que c'était au dépens du reste de ma personne. Les marques les moins équivoques de ce changement furent une très grande sécheresse de poitrine, une égale difficulté de respirer, une chaleur considérable entre les deux épaules, et une froideur mortelle à toutes les extrémités du corps... Je pris toutes ces indices pour des symptômes d'une mort certaine et inévitable, de sorte que sans songer à l'art d'Esculape, je ne pensai plus qu'aux affaires de ma conscience...»

«Lorsque le jour parut, m'étant aperçu que les symptômes... étaient à peu près au même état... je commençai à rassurer contre les frayeurs du trépas, mais vers le midi, j'eus une nouvelle alarme en sentant que le sommet de ma tête devenait aussi froid que si on y eut posé un morceau de glace et que les cheveux qui couvraient cette partie refroidie tombaient par poignées. J'en fus quitte pour être chauve et de déposer le reste de mes cheveux pour prendre la perruque. Je tombai insensiblement dans une langueur où la 
faiblesse du corps avait beaucoup plus de part que celle du cœur. La difficulté que j'avais de respirer me mit hors d'état de m'appliquer comme auparavant et j'éprouvai à l'égard de l'amour, la cigue avait produit autant d'effet sur lui que l'on en pourrait produire sur le feu.»

Invité par son Mécène ${ }^{19}$ à Plombières, «je m'appliquai à dissiper le froid dont j'étais glacé aux extrémités du corps en me plongeant deux fois par jours dans l'eau chaude, et y demeurant le plus longtemps qu'il m'était possible, surtout lorsque j'eus oui discourir un vieux médecin de Basle en Suisse sur la nature de quelques plantes venimeuses, ou parlant de la cigue il avait déclaré que le suc de cette plante était un poison froid qui faisait le même effet sur le sang que la présure sur le lait. La décision de cette esculape m'occasionna une grande quantité de brulantes immersions dans le dessein de rétablir mon sang dans sa première fluidité. La chaleur des bains y contribua beaucoup, cependant cette incommodité ne s'évanouit entièrement que plus de deux ans après, mais à l'égard de la sécheresse de poitrine, j'en ai toujours ressenti les fâcheux effets et il y a apparence qu'ils dureront jusqu'à la fin de mes jours.»

\section{Le diagnostic}

A une élève de H. Schadewaldt, Adelheid Giedke ${ }^{17}$ revient le mérite d'avoir présenté l'étude peut-être la plus pertinente, la plus complète aussi, de la maladie d'amour dans l'histoire de la médecine.

Partant de Sappho et, sur le plan médical, de l'ancienne observation attribuée à Erasistrate, d'Hippocrate et de Galien, l'auteur étudie les byzantins Oribase et Paul d'Egine pour lesquels l'amour est un facteur pathogénique en soi et qui précisent les symptômes décrits par les Grecs. Avicenne élargit la connaissance de l'amour excessif et en développe une théorie basée sur l'humorisme. A partir de la Renaissance on a la tendance de voir les symptômes plutôt en relation avec d'autres maladies (fièvres, organopathies, maladies mentales). Au temps des Lumières, l'éromanie de De Baillou ${ }^{4}$, l'érotomanie de Boissier de Sauvages est sujette à une classification nosologique plus rigoureuse. Elle revêt le caractère d'une manie partielle, la plupart des facultés intellectuelles et aussi le sens moral restant intactes.

La multitude des synonymes du «hereos» du moyen âge jusqu'à la «monomanie érotique» du XIX ${ }^{\mathrm{e}}$ siècle (Esquirol 1818, Monneret 1857) ne doit pas tromper. Le délire de Duval aurait trouvé un classement approprié à n'importe quelle époque, tellement était rectiligne le parcours qu'avait pris la médecine; les recherches étiologiques n’y ont apporté que des modifications insignifiantes.

Pour le médecin du XVIII ${ }^{\mathrm{e}}$ siècle, nourri d'une longue tradition aussi bien médicale que littéraire, le diagnostic de l'érotomanie eut été aisé, quoiqu'il ne 
fût pas facile de trouver toujours une limite entre réactions normales et pathologiques. "Che di Venera avea sentito il tosco», comme disait Dante ${ }^{10}$, était un malade potentiel. Pour certains auteurs toute manifestation amoureuse, non seulement l'amour excessif, relèverait donc de la pathologie et le refroidissement marquerait le retour à l'équilibre, à une placide indifférence (ataraxie).

On peut s'étonner que Duval dans son auto-analyse et ses recherches livresques n'ait pas consulté Lucrèce ${ }^{21}$ qui avait décrit une situation semblable (Livre IV, 1067 s.):

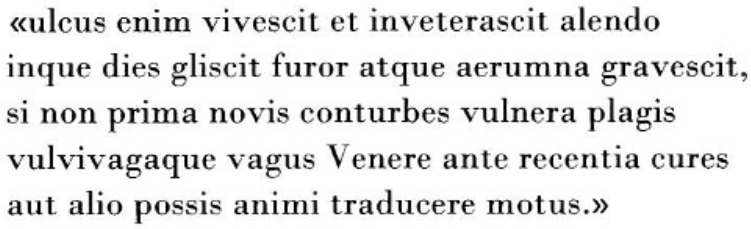

Mais cet auteur tout présent au XVIII ${ }^{\mathrm{e}}$ siècle, l'était peut-être moins dans certains milieux de prédominance ecclésiastique, manquant ainsi à l'érudition naissante de Duval, autant que le poète des Remèdes d'amour.

La situation physiologique particulière de l'adolescent évoquée par Lucrèce (IV, 1037-38)

«sollicitatur in nobis (quod diximus ante)

semen, adulta aetas cum primum roborat artus»,

était aussi bien connu des auteurs du moyen âge, et il est presque certain que des restes de leur savoir étaient conservés dans la médecine populaire. On devrait penser p.ex. aux «Concordanciae» de Jean de Saint-Amand, enseignant la médecine à Paris au XIII ${ }^{\mathrm{e}}$ siècle ${ }^{24}$ et dont les sentences reprenant la diététique de Galien et très répandues à leur temps, ont été rééditées par Julius Leopold Pagel ${ }^{25}$ : «In juvenibus est sanguis multus, virtus fortis, caliditas multa (p. 156); Humores et compositio et natura mutant actionem animae (p.14); Si sperma aggregetur in corpore et non expellatur per coitum generantur ex eo aegritudines malae» (p. 54). Même un article «amor hereos» s'y trouve. Mais il ne faut pas oublier que Duval appartenait à une jeunesse studieuse, dont l'intérêt principal était l'histoire et l'interprétation des textes sacrés et qui ignorait les données de la médecine, si l'on excepte son côté théorique représenté par la physiologie de Descartes.

Le problème de l'adolescence demeurait entier au XVIII ${ }^{\mathrm{e}}$ siècle quand Buffon (1707-1788) publia l'Addition (1774) à l'article «De la Puberté». ${ }^{8}$ Ce 
mémoire pourrait être mis en parallèle avec certains événements vécus par Duval. Dans son introduction, toujours soucieux de répandre la clarté, Buffon écrit: «... quand on s'obstine à contrarier la nature, il en arrive souvent des funestes effets, dont il est bon de donner quelques exemples».

Que signifie l'érotomanie pour la médecine du XVIII ${ }^{\mathrm{e}}$ siècle? La célèbre thèse $(1724)^{5}$ (n 6) de François Boissier de Sauvages est plutôt regardée comme un exercise poétique en honneur d'Ovide, d'Horace et de Virgile, et ajoutant peu à la grande renommée médicale de son auteur ${ }^{14}$. Il serait pourtant injuste de méconnaître les vertus de ce stimulant travail et de négliger l'esprit d'observation qui s'y manifeste. Dans sa «Nosologie méthodique» ${ }^{6}$ Boissier de Sauvages a subtilement analysé les «maladies morales», «celles qui dépendent des fonctions erronées de l'âme, comme de leur cause prochaine; du déréglement de l'imagination, par exemple, de l'appétit, de la volupté et du jugement»(III, 593s.). «... les hypochondriaques, les mélancholiques, qui ne savent pas modérer les affections de leur esprit, à cause de leur sensibilité jointe à leur faiblesse, mènent une vie fort malheureuse.» Ceux qui sont dominés «par la passion de l'amour»sont attaqués de pâles couleurs, ne dorment ni ne mangent, et se consument. «Ceux qui s'appliquent trop à l'étude, affaiblissent leur corps, et tombent aisément dans des maladies de l'esprit, à moins qu'ils ne se reposent par intervalles, et ne se récréent à la promenade, à la campagne ou avec leurs amis: ils perdent le repos et l'appétit, deviennent mélancholiques, maigres et ressentent d'autres maux que le sommeil, les bains et la diète guérissent plus sûrement que les médicaments.» Ainsi la conclusion de la thèse de 1724 «ergo amor est medicabilis herbis» est, sinon contredite, du moins modifiée par l'introduction d'agents non-médicamenteux dans la thérapeutique.

Boissier de Sauvages dans son Vocabulaire de la Nosologie (éd.1771) donne la définition: «Amour déréglé, Mélancolie amoureuse, Erotomanie. Cette affection diffère du satyriase, et de la nymphomanie, en ce que les malades ne désirent point uniquement la possession charnelle de l'objet aimé; mais qu'ils le respectent au contraire comme un Dieu» (voir l'expérience de Duval). Boissier renvoie aux conseils d'Ovide sur cette affection. Dans sa «Nosologie de la pathologie» ${ }^{7}$ il résume: «Erotomanie, amour insensé, erotomania. C'est un désir violent, mais honnête des plaisirs de l'amour.»

Pour William Cullen (1712-1790) ${ }^{9}$ l'érotomanie est intégrée dans le complexe de la mélancholie. Il est de même pour Gerard van Swieten $(1700-1772)^{36}(\mathbf{n} 7)$. Cette tendance caractérise le siècle entier. Cullen, 
traduit par Bosquillon (1744-1816), précise: «On considère communément la mélancholie comme une folie partielle, et je l'ai définie comme telle dans ma nosologie; mais aujourd'hui je doute que cette définition soit bien exacte. Par folie partielle, j'entends un jugement faux et erroné sur un objet particulier, et sur ce qui y a rapport; quoique le malade juge de tout autre objet comme le commun des hommes. Il y a certainement eu de ces exemples de folie; mais je pense que l'on en a peu observé où la folie partielle fût strictement limitée.»

Bosquillon commente (d'après la nosologie de Cullen): «La mélancolie varie en raison des objets sur lesquels le malade délire. Ainsi: La mélancolie consiste dans un amour excessif... Telle est l'érotomanie ou la mélancolie amoureuse. Cette espèce de mélancolie diffère du satyriasis et de la nymphomanie, en ce que ceux qui en sont affectés ne désirent point jouir impudemment des faveurs de l'objet de leur amour; mais au contraire ils le révèrent comme une divinité... ils ne dorment pas, refusent de prendre des aliments, et abandonnent toutes leurs occupations.» Le commentateur érudit et lettré cite les cas de Lucrèce, du Tasse et d'Aristote, dont De Baillou (4) avait rapporté le mot: «amor insanus turpior ira» (n 8).

Abstraction faite de quelques solidistes indécis, la conception humorale était celle de la médecine du XVIII ${ }^{\mathrm{e}}$ siècle et "par definitionem» l'excédent d'une des quatre humeurs était la cause pathogénique de la mélancolie. Par conséquent le délire amoureux avait les mêmes causes naturelles, un substrat matériel que l'on pourrait changer, soustraire. Starobinski dans son essai «L'épée d'Ajax» ${ }^{33}$ a décrit le processus qui depuis l'Antiquité n'avait pas perdu sa force de conviction: «La mélancolie, humeur noire, exerce sa contrainte en sa qualité d'agent physique. Cette substance peut entrer en effervescence, mais la chaleur ainsi produite fait rapidement place au froid. Dans le comportement du mélancolique, cette variabilité s'exprime par l'alternance de la fureur et de l'abattement, de l'agitation et de la tristesse. Ce n'est plus la colère du dieu offensé qui l'aiguillonne et l'égare, mais la loi du corps. Le délire (d'Ajax) n'est plus qu'un cas de dyscrasie mélancolique où la colère, l'égarement, puis le désespoir suicidaire s'enchaînent conformément à l'attente du savoir médical.»

L'érotomanie devrait donc s'offrir à un traitement physique ou chimique, susceptibles d'éliminer, de supprimer les causes naturelles des troubles, causes transportées par le sang et se manifestant essentiellement par l'organe producteur du liquide séminal (n9). 


\section{Le traitement}

Dans la troisième partie de ses Mémoires ${ }^{19}$, Duval avait fait l'aveu: «... en parcourant l'immense collection des Pères (n 10), je fus très fâché quand St.Brunon d'Ast m'eut appris que le Diable avait l'audace d'établir son domicile dans certaines régions du corps humain... J'avais peine à concevoir pourquoi cet esprit de ténèbres s'y plaisait plus qu'ailleurs et pourquoi il s'en attribuait la Direction. J'eûs aussi le malheur de faire un peu trop d'attention à un passage de St. Jérôme où il est dit que les Hiérophantes... se servaient de jus de cigue pour acquérir dans le Sacerdoce une qualité tout à fait opposée à celle qui est requise dans la vie conjugale». On a vu (supra) «quel effet ce passage produisit sur mon esprit et l'étrange abus que j'en fis en péril de ma vie, pour me délivrer d'une passion qui ne s'accordait point avec la tranquillité qu'exigeaient mes études» (n 11).

Dans le «traitement de l'aphrodisie» qui soulève «une question de moralité», le médecin légiste strasbourgeois Gabriel Tourdes (1810-1900) ${ }^{34}$ rappelle que (selon J.P.Frank) «Galien a préconisé la castration; il cite les vers de Lucrèce: Et graviter partim metuentes limina lethi... qui consacrent cette méthode, il semble lui-même disposé à l'admettre pour les fous érotomanes». Pierre-Adolphe Piorry (1794-1879) ${ }^{26}$ sous la rubrique «anomopsychismies: érotomanie, thérapisme» constate: «on voit... l'amour excessif souvent cesser lors de la possession de l'objet aimé (à tel point que celui-ci devient parfois tout à fait indifférent) ou alors que les organes génitaux sont détruits... Abailard après son malheur devient dévot et raisonnable, Héloise continue à se livrer aux transports de sa passion alors que son amant est devenu eunuque».

Origène (v. 185-v.254) en se faisant émasculer (v.203) avait en quelque sorte montré le chemin, même s'il eût été la victime d'une interprétation

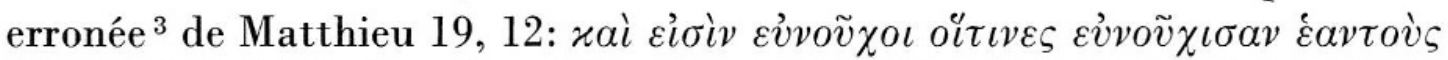

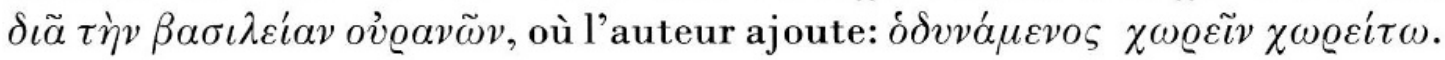
Jérôme a traduit: «et sunt eunuchi qui seipsos castraverunt propter regnum caelorum. Qui potest capere capiat.»

Selon Clément d'Alexandrie (v.150-v.215), précepteur d'Origène, la chute des anges aurait été provoquée par leur concupiscence qu'ils ne savaient pas surmonter ${ }^{28}$. Leur union avec des femmes aurait produit une classe de démons qui désormais se trouvent dans la compagnie du diable. «Sic eos Diabolus ex angelis Dei fecit satellites ac ministros» comme assure Lactance (v.260-v.325) ${ }^{20}$. Ils détériorent, ils corrompent la nature 
humaine: «Qui quoniam sunt spiritus tenues, et incomprehensibiles, insinuant se corporibus hominum, et occulte in visceribus operati valetudinem vitiant, morbos citant, somniis animos terrent, mentes furoribus quatiunt: ut homines his malis cogant ad eorum auxilia decurrere.»

Pour un monde qui inclinait plutôt vers la philosophie austère de Plutarque «non posse suaviter vivi secundum Epicurum», ${ }^{1}$ la plupart des Remedia amoris d'Ovide ${ }^{22,23}$ étaient proscrits, bien qu'il ait fait la promesse (selon un traducteur anonyme du $\mathrm{XVIII}^{\mathrm{e}}$ ):

«Vous, que d'un fol amour égarent les erreurs,

Je viens vous affranchir de toutes ses horreurs.

Dans vos premiers soupirs, je vous servais de guide,

Pour ne plus soupirer, suivez encore Ovide.»

Pourtant, deux conseils donnés par Ovide, avait suivi Duval presque instinctivement, celui de la distraction («fuyez la solitude», sans succès) et celui du médicament supprimant ses désirs:

«Corpora vix ferro quaedam sanantur acuto:

Auxilium multis succus et herba fuit.»

Bien que le XVIII ${ }^{\mathrm{e}}$ siècle eût l'habitude des anaphrodisiaques ${ }^{29}$ et en utilisât un bon nombre (d'efficacité douteuse), Duval, en suivant l'indice de St.Jérôme, choisit un qu'il croyait capable d'effectuer une castration adoucie: le plus toxique, la Ciguë.

Les Romains avaient condamné son usage, autant que d'autres «pocula odii» et certains aphrodisiaques («Declamationes» d'un Senatus consultum du $1^{\text {er }}$ siècle ap. J.-C.) ${ }^{30}$. Pline (23-79) a insisté sur la haute toxicité de cette plante $^{27}$ : «Cicuta quoque venenum est, publica Atheniensium poena invisa, ad multa tamen usus non omittendi... Semini et foliis refrigeratoria vis: quos enecat, incipiunt algere ab extremitatibus corporis. Remedio est, priusquam perveniat ad vitalia, vini natura excalfactoria. Sed in vino pota, irremediabilis existimatur (Lib. XXV, XCV). Au XVIII ${ }^{\mathrm{e}}$ siècle Jacques-Christophe Valmont de Bomare (1731-1807) donne une bonne déscription des différentes espèces. La Ciguë «est un poison froid, narcotique qui s'attache au genre nerveux et agit sur la masse du sang en dissolvant et non en coagulant» (Mémoires de la Société Royale de Montpellier). L'usage comme remède «était tout-à-fait tombé dans l'oubli», jusqu'à son renouvellement (1760) par le Viennois Anton Stoerck (1731-1803).

Bien qu'inexprimé, l'idée d'une action antiaphrodisiaque de la Ciguë reste latente. Joseph-François Delioux de Savignac (1812-1876) relate son 
effet sur l'appareil génital ${ }^{12}$ : «On a at tribué à la ciguë le pouvoir d'atrophier les mamelles, les testicules; d'entraîner l'impuissance virile. Sans nier ces effets d'une manière absolue, on ne doit les accepter que sous réserve, car quelques auteurs ont considéré au contraire la ciguë comme aphrodisiaque. La propriété de tarir la sécrétion lactée paraît plus réelle.»

Malgré ce scepticisme, Jean-Baptiste Fonssagrives (1823-1884) ${ }^{16}$ confirme son application comme anaphrodisiaque en précisant les indications: priapisme, nymphomanie. Et dans la Matière médicale rimée ${ }^{37}$ du médecin belge Jules Vindevogel (1897) on peut lire sous «Cigue et Cicutine, Conéine, Conicine» les vers:

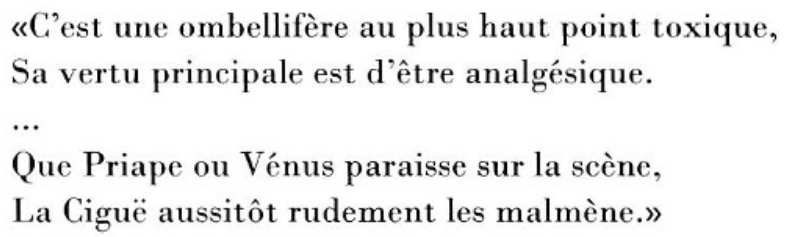

Après avoir pris «le mauvais et dangereux parti» de faire à sa tête, Duval se souvint de l'art médical ${ }^{19}$ : «Quelque médecin ou botaniste aurait pu me tirer d'embarras en me dirigeant dans une opération aussi scabreuse et aussi délicate, mais il ne me vint même pas dans la pensée d'en consulter un.» Et pourtant, la meilleure étude que l'on puisse s'imaginer à cette époque, aurait pu être à sa disposition: l'histoire de la Ciguë aquatique (1679) ${ }^{39}$ par Joh. Jac. Wepfer (1620-1695), medicus scaphusianus. Une bonne partie de ce que Duval avait souffert y est décrit: «Frigiditas extremorum; gravitas motus corporis; suffocatio et strictura anhelitus; permixtio rationis; caligo oculorum subit seu somnus profundus; singultus, dolor stomachi; color labiorum citrinus vel viridis; corporis plumbeus stupor membrorum; durities pulsus; quies venarum pulsatilium et post quietem illam Mors, nisi curatio acceleretur» (p.41). Il semble peu probable que Wepfer, ou un médecin de son temps, aurait pu ou voulu assister Duval dans son dessein. A un rétablissement infiniment lent avait présidé, inconsciemment, le conseil de Jean de Saint-Amand ${ }^{25}$ du XIII ${ }^{\mathrm{e}}$ siècle: «Melancholia quae ex colera nigra in cerebro curatur per balneum et cibos humidos boni chymi» (p. 191).

Certes, ce rapport essentiellement médico-historique laisse maintes questions sans réponse, aussi celle de l'intention de cette autobiographie, que l'on peut se poser malgré l'analyse très poussée par Goulemot ${ }^{19}$. Faudrait-il voir dans cet auteur une sorte de conscience sociale restée muette à son époque? Car, les extraits manuscrits de ses Mémoires, confiés à quelques amis, passaient presque inaperçus. Formaient-ils un travail préparatoire 
pour «Les aventures de l'étourderie», roman philosophique perdu, si l'on doit croire aux biographes (n 1)? Plus proche de la vérité est resté sans doute Deleyre (supra).

Ecrivant pour lui-même, Duval avait actualisé le processus de sa libération personnelle, de sa montée vers un idéal. Voltaire, connaissant son curriculum vitae, a eu la bonne intuition de placer Duval à la tête de son article encyclopédique «Astronomie» ${ }^{38}$ comme modèle pour une jeunesse avide de savoir et ne disposant que de ses dons intellectuels pour se réaliser. Juste cet épisode du garçon berger observant les étoiles, illustre la page de titre de la première édition de ses Mémoires ${ }^{18}$. Ce n'est peut-être qu'un aspect. Existe-t-il une affinité plus profonde entre Duval et Rousseau, comme l'a suggéré Goulemot? Peut-être l'application de la méthode si brillamment utilisée par Jean Starobinski pour étudier les problèmes de l'autobiographie chez Jean-Jacques Rousseau ${ }^{31}$ pourrait donner de nouvelles lumières.

\section{Notes}

(n 1) «Victor von Koch» d'après la note bibliographique de Constant von Wurzbach: Duval, Valentin Jamerai (Direktor des kais. Münz-Cabinetes, geb.zu Artonay in der Champagne, 12.Jän.1695, gest.zu Wien 3.Nov.1775) in: Biographisches Lexikon des Kaiserthums Österreich 3.T.p.401-403, Wien 1858. L'édition des Euvres (1784) donne les initiales F.A. de K...

(n 2) Dessinateur de «vues» et graveur, regardé comme le Canaletto viennois; «réaliste» très estimé à son époque.

(n3) Aussi la courte note biobibliographique dans le Lexique de v. Wurzbach (supra n 1), note que je dois au Professeur Wyklicky (Vienne), rapporte cette histoire.

(n4) En principe, l'orthographe des citations a été adaptée aux règles actuelles.

(n5) Jovinien enseigna que la virginité ne devait pas avoir la préférence devant l'union conjugale, et que le baptisé était hors toute atteinte du diable ${ }^{3}$.

(n 6) Elle fut pour la première fois imprimée en $1854^{13}$.

(n 7) Hermann Boerhaave (1668-1738) regarde la mélancolie comme l'effet de l'atrabile $=$ «succus melancholicus» (Aphor. 1090 et 1092) ${ }^{36}$. La distinction d'Anne-Charles Lorry (1726-1783) entre mélancolies spasmodique, nerveuse et humorale (1765) ${ }^{17}$, un essai de différencier, n'est pas nécessairement une objection contre la généralisation de la théorie humorale.

(n8) Selon Ernst von Feuchtersleben (1806-1849) l'érotomanie laisse souvent apparaître une composante religieuse, dont le malade n'a pas conscience. Cette forme mixte aurait été rapportée par Johann Georg Zimmermann (1728-1795) dans son livre sur la solitude ${ }^{15}$.

(n9) Cette liqueur est «préparée et séparée du sang dans les testicules» (Férapied Dufieu, Dictionnaire raisonné d'Anatomie et de Physiologie, Paris 1766). 
(n 10) Maxima Bibliotheca Veterum Patrum... 27 volumes Lyon 1677, selon Goulemot ${ }^{19}$.

(n11) Dans la suite, Duval se livre à une acerbe critique des interprêtes, commentateurs et expositeurs de la Ste Ecriture en comparant leur attitude à l'éloquente chicane des avocats: «J'aurais souhaité que la plupart de leurs décisions eussent été un peu moins impérieuses et qu-ils n'eussent pas si souvent confondus les Lueurs de la Probabilité avec les Clartés de la Démonstration.»

\section{Bibliographie}

${ }^{1}$ Adam, Hella: Plutarchs Schrift non posse suaviter vivi secundum Epicurum. Grüner, Amsterdam 1974.

${ }^{2}$ Adhémar, Jean: La gravure originale au $18^{\mathrm{e}}$ siècle. Somogy, Paris 1963

${ }^{3}$ Altaner, Berthold: Patrologie. Herder, Freiburg 1938

4 Ballonius (de Baillou), Guillaume: De virginum et mulierum morbis (Cap. IX de insano virginum amore) in Opera omnia medica (T. IV) ed. Jacques Thévart, 4 vol., Venetiis 1734

${ }_{5}^{5}$ Boissier de Sauvages, François: Dissertatio medica atque ludicra de amore: Utrum sit amor medicabilis herbis (1724). Monspeliensis Hippocrates 6, No. 20 (1963) 10-16

${ }^{6}$ Boissier de Sauvages, François: Nosologie méthodique. Ed.P.-F. Nicolas, 3 vol., Hérissant, Paris 1770-1771

${ }^{7}$ Boissier de Sauvages; François: CEuvres diverses. 2 vol.(T. I, 334). Costard, Paris 1771

${ }^{8}$ Buffon, Georges Louis Leclerc de: Histoire naturelle. Edition Lacépède: Matières générales, 24 vol. (T. XX, Histoire de l'homme). Didot, Paris 1799

${ }^{9}$ Cullen, (William) Guillaume: Eléments de Médecine-Pratique traduits par EdouardFrançois-Marie Bosquillon, 2 vol. Barois et Méquignon, Paris 1787

${ }^{10}$ Dante Alighieri, Durante: Die göttliche Komödie, italienisch und deutsch, übers.u.komment. von Hermann Gmelin, 6 Bde. Klett, Stuttgart 1949-1957

${ }^{11}$ Delioux de Savignac, Joseph-François-Jacques-Augustin: Art. «Cigue» in Dict.Sci. Méd. (Dechambre) T. XVII, 238-264. Masson, Paris 1875

${ }^{13}$ D'Hombres-Firmas, Louis-Augustin: La thèse de Boissier de Sauvages. Monspeliensis Hippocrates 6, No.20 (1963) p. 9

${ }^{14}$ Dulieu, Louis: Bref aperçu sur l'œuvre de François Boissier de Sauvages. Monspeliensis Hippocrates 6, No.20 (1963) 4-8

15 Feuchtersleben, Ernst von: Lehrbuch der ärztlichen Seelenkunde. Gerold, Wien 1845

${ }^{16}$ Fonssagrives, Jean-Baptiste: Traité de Matière médicale. Delahaye et Lecrosnier, Paris 1885

${ }^{17}$ Giedke, Adelheid: Die Liebeskrankheit in der Geschichte der Medizin. Diss. Med. Fak. Düsseldorf, 1983

18 Jamerey-Duval, Valentin: CEuvres de Valentin Jamerai Duval, précédées des Mémoires sur sa vie. Avec figures, 2 vol. A.S. Pétersbourg et se vend à Strasbourg chez J. G. Treuttel 1784

${ }^{19}$ Jamerey-Duval, Valentin: Mémoires, Enfance et éducation d'un paysan au XVIII ${ }^{\mathbf{e}}$ siècle. Avant-propos, introduction, notes et annexes par Jean Marie Goulemot. Le Sycomore, Paris 1981

${ }^{20}$ Lactantius Firmianus, Lucius Caecilius: Divinarum institutionum (Lib. II, De origine erroris, Cap. XIV) in Opera omnia. Ed. Christoph August Heumann. Gottingae 1736 
${ }^{21}$ Lucretius Carus, Titus: De rerum natura libri sex. Ed. Michael Maittaire. Tonson, Watts, Londini 1713

22 Ovide: Les œuvres galantes et amoureuses, contenant L'art d'aimer, le remède d'amour etc. (A Cythère, au dépens du loisir) 1774

${ }^{23}$ Ovidius Naso, Publius: Opera quae supersunt. 3 vol. (T.I: Remedia amoris, T.II: Metamorphoseon). Barbou, Paris 1762

${ }^{24}$ Pagel, Julius Leopold: Die Areolae des Johannes de Sancto Amando (13. Jahrhundert) nach Handschriften der Königlichen Bibliotheken zu Berlin und Erfurt zum ersten Male herausgegeben. Reimer, Berlin 1893

25 Pagel, Julius Leopold: Die Concordanciae des Johannes de Sancto Amando nach einer Berliner und zwei Erfurter Handschriften zum ersten Male herausgegeben. Reimer, Berlin 1894

${ }^{26}$ Piorry, Pierre-Adolphe: Traité de médecine pratique. 9 vol. (T. VIII). Baillière, Paris $1842-1851$

${ }^{27}$ Plinius Secundus, Caius: Historiae naturalis libri XXXVII. 8 vol. (T.VI). Panckoucke, Paris 1836

${ }^{28}$ Roskoff, Gustav: Geschichte des Teufels. 2 vol. Brockhaus, Leipzig 1869

${ }^{29}$ Rudolph, Gerhard: Médicaments à action neurotrope au XVIII ${ }^{\mathrm{e}}$ siècle. Actes XXVIII Congrès international d'Histoire de la Médecine, Vol. II, 239-243, Paris 1982

30 Schelenz, Hermann: Geschichte der Pharmazie (réimpression de l'édition Berlin 1904). Olms, Hildesheim 1962

31 Starobinski, Jean: Jean-Jacques Rousseau - La transparence et l'obstacle. Plon, Paris 1957

32 Starobinski, Jean: L'invention de la liberté 1700-1789. Ed.all. «Die Erfindung der Freiheit» trad. H.Staub. Skira, Genève 1964

${ }^{33}$ Starobinski, Jean: Trois fureurs, Essais. Gallimard, Paris 1974

34 Tourdes, Gabriel: «Aphrodisie et Anaphrodisiaques» in Dict.Sci. Méd. (Dechambre) T.5, 652-666, Masson, Paris 1866

35 Valmont de Bomare, Jacques-Christophe: Dictionnaire raisonné universel d'Histoire naturelle... avec l'Histoire et la déscription des Drogues simples tirées des trois règnes.

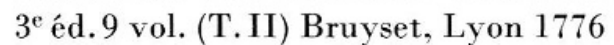

36 Van Swieten, Gerard: Commentarii in Hermanni Boerhaave Aphorismos de cognoscendis et curandis morbis. 5 vol. (T. III). Cavelier, Paris 1771

37 Vindevogel, Jules: (Triptique de la littérature médicale) La matière médicale définie. Lombaerts, Bruxelles 1897

38 Voltaire, François Marie Arouet de: Dictionnaire philosophique (I) in CEvres complètes. Nouvelle édition. 60 vol. (T.XXXIII, Art.Astronomie). Carez, Thomine et Fortic. Paris 1820

${ }^{39}$ Wepfer, Johann Jacob: Cicutae aquaticae historia et noxae. König, Basileae 1679 


\section{Summary}

In his Mémoires Valentin Jamerey-Duval (a former uncultivated sheperds boy who, by the acquisition of an enormous erudition, became director of the imperial numismatic cabinet at the time of Maria-Theresia in Vienna) relates an access of excessive love arriving in his adolescence which he tried to suppress by medicamentous castration with cicuta, following an allusion given by St.Jerome. The historical and medical background of Erotomania is analysed. Its incorporation in the complex of Melancholia in the XVIIIth century would justify a pure somatic treatment for instance with anaphrodisiac drugs.

Prof. Dr.med. Gerhard Rudolph

Universität Kiel

Bülow-Straße 16

D-2300 Kiel 